\title{
CLUSTERING ON GRASSMANN MANIFOLDS VIA KERNEL EMBEDDING WITH APPLICATION TO ACTION ANALYSIS
}

\author{
Sareh Shirazi, Mehrtash T. Harandi, Conrad Sanderson, Azadeh Alavi, Brian C. Lovell \\ NICTA, PO Box 6020, St Lucia, QLD 4067, Australia \\ University of Queensland, School of ITEE, QLD 4072, Australia
}

\begin{abstract}
With the aim of improving the clustering of data (such as image sequences) lying on Grassmann manifolds, we propose to embed the manifolds into Reproducing Kernel Hilbert Spaces. To this end, we define a measure of cluster distortion and embed the manifolds such that the distortion is minimised. We show that the optimal solution is a generalised eigenvalue problem that can be solved very efficiently. Experiments on several clustering tasks (including human action clustering) show that in comparison to the recent intrinsic Grassmann $k$-means algorithm, the proposed approach obtains notable improvements in clustering accuracy, while also being several orders of magnitude faster.
\end{abstract}

Index Terms - Grassmann manifolds, kernels, clustering, Reproducing Kernel Hilbert Spaces, action analysis.

\section{INTRODUCTION}

Reformulating computer vision problems over non-Euclidean spaces (such as Riemannian manifolds) has recently received growing attention $[5,10,13,14,17,18]$. This includes a wide range of applications such as face recognition [18], pedestrian detection [14], action recognition [11], tracking [19] and motion segmentation [9]. As video analysis can be accomplished with the help of subspaces modelling, Grassmann manifolds (a special type of Riemannian manifolds) play a significant role in these recent advances $[1,5,7,18]$.

Broadly speaking, inference methods over manifolds can be classified into intrinsic and extrinsic approaches. Intrinsic approaches are entirely based on the manifolds themselves and do not rely on any embedding, while extrinsic methods involve embedding the manifold into an Euclidean space (eg. via tangent spaces). As such, extrinsic methods inherit the Euclidean metrics, which leads to a simpler form of the original inference problem. While clustering (a form of inference) over Euclidean spaces has a long history [6], clustering over Riemannian manifolds (and especially Grassmann manifolds) has received very little attention.

To the best of our knowledge, only three works have addressed the problem of clustering over Grassmann manifolds.

\footnotetext{
Acknowledgements: NICTA is funded by the Australian Government as represented by the Department of Broadband, Communications and the Digital Economy, as well as the Australian Research Council via the ICT Centre of Excellence program.
}

Subbarao and Meer [17] proposed an extrinsic method where the Mean Shift (MS) algorithm [2] is extended to Grassmann manifolds. More specifically, the manifold tangent space (intuitively a space of directional velocity, which is also an Euclidean space) is used to compute the mean shift. Cetingul and Vidal [1] developed an intrinsic MS on Stiefel and Grassmann manifolds. The main difference between extrinsic and intrinsic MS is that in the latter kernel density functions are computed through optimisation over manifolds.

Recently, Turaga et al. [18] devised a form of semiintrinsic $k$-means clustering on Grassmann manifolds through the use of the Karcher mean [8]. The Karcher mean is an iterative approach to calculate the mean of a set of points on a Riemannian manifold. More specifically, mean $\overline{\boldsymbol{m}}$ of a set of Riemannian points $\left\{\boldsymbol{X}_{i}\right\}_{i=1}^{n}$, can be obtained as $\overline{\boldsymbol{m}}=\operatorname{argmin}_{\boldsymbol{m}} \sum_{i} d_{\mathcal{R}}^{2}\left(\boldsymbol{m}, \boldsymbol{X}_{i}\right)$, where $d_{\mathcal{R}}$ is a Riemannian metric $[14,17]$. This minimisation problem is solved by iteratively moving from manifold to tangent spaces and vice versa. This can be quite time consuming, especially for computer vision applications where the dimensionality of data can be high. More importantly, like any other intrinsic approach, this method is confined to the nature of the distribution of points on the manifold. This is restrictive since the scattering pattern of the Grassmann points might not be optimal for clustering purposes. A related phenomenon has been noted by Dhillon et al. [3] in the context of clustering in Euclidean spaces.

In this paper, we propose an extrinsic clustering approach on Grassmann manifolds and show that it outperforms the intrinsic Grassmann $k$-means algorithm [18], while also being considerably faster. To this end, we define a measure of clustering distortion and embed the Grassmann manifold into a Hilbert space [15] such that the measure of distortion is minimised. In essence, this metric measures the compactness of each cluster, ie., how closely the points are scattered around the mean of the cluster. We show that the manifold embedding problem is a generalised eigenvalue problem that can be solved efficiently [3]. As such, traversing from the manifold to its tangent spaces is avoided, leading to a considerably lower computational complexity when compared to intrinsic Grassmann $k$-means.

We continue this paper as follows. Section 2 briefly describes the geometry of Grassmann manifolds. The proposed clustering method is presented in Section 3, with its performance compared in Section 4. The main findings and possible future directions are summarised in Section 5. 


\section{BACKGROUND}

Formally, a manifold is a topological space that is locally similar to an Euclidean space. A Grassmann manifold can be viewed as a quotient space of the special orthogonal group $S O(r)$ and is defined as the space of all $p$-dimensional subspaces of $\mathbb{R}^{r}$.

Since Grassmann manifolds are curved, the distance between two points is given by the length of curves between them. The curve with the minimum length is known as the geodesic and the length of the geodesic is the geodesic distance.

Due to the specific geometrical properties of Grassmann manifolds, Grassmann kernels have been developed to embed the Grassmann space into Reproducing Kernel Hilbert Spaces (RKHS) [5]. Let $\mathcal{G}_{r, p}$ be a Grassmann manifold and $k: \mathcal{G}_{r, p} \times \mathcal{G}_{r, p} \rightarrow \mathbb{R}$ be a symmetric real-valued function. $k$ is a Grassmann kernel if it satisfies two conditions, namely being positive definite and well-defined. More specifically, a function $k$ is a Grassmann kernel if:

1. $\sum_{i, j} c_{i} c_{j} k\left(\boldsymbol{X}_{i}, \boldsymbol{X}_{j}\right) \geqslant 0$ for all $\boldsymbol{X}_{i} \in \mathcal{G}_{r, p}$ and $c_{i} \in \mathbb{R}$

2. $k\left(\boldsymbol{X}_{i}, \boldsymbol{X}_{j}\right)=k\left(\boldsymbol{X}_{i} \boldsymbol{O}_{i}, \boldsymbol{X}_{j} \boldsymbol{O}_{j}\right)$ for all $\boldsymbol{X}_{i}, \boldsymbol{X}_{j} \in \mathcal{G}_{r, p}$, where $\boldsymbol{O}_{i}, \boldsymbol{O}_{j} \in Q(p)$ are orthonormal matrices of order $p$.

Among the various Grassmann kernels [5], we have used the projection kernel as it has been previously shown to work well with video data [5]. The kernel is defined as:

$$
k_{P}\left(\boldsymbol{X}_{i}, \boldsymbol{X}_{j}\right)=\left\|\boldsymbol{X}_{i}^{T} \boldsymbol{X}_{j}\right\|_{F}^{2}=\operatorname{tr}\left\{\left(\boldsymbol{X}_{i} \boldsymbol{X}_{i}^{T}\right)\left(\boldsymbol{X}_{j} \boldsymbol{X}_{j}^{T}\right)\right\}
$$

\section{PROPOSED APPROACH}

Given a set of Grassmann points $\left\{\boldsymbol{X}_{i}\right\}_{i=1}^{n} ; \boldsymbol{X}_{i} \in \mathcal{G}_{r, p}$, the problem at hand is to group the points into $k$ disjoint clusters. The similarity measure for clustering should consider the geometry of the manifold as much as possible. To this end, we first embed the Grassmann manifold into RKHS space by virtue of a Grassmann kernel.

Assume that the set $\left\{\phi\left(\boldsymbol{X}_{i}\right)\right\}_{i=1}^{n}$ (with $\phi\left(\boldsymbol{X}_{i}\right) \in \mathbb{R}^{D}$ ) contains the implicit representation of Grassmann points in RKHS. Inspired by kernel $k$-means in Euclidean space [3], we define $d\left(C_{j}\right)=\sum_{i \in C_{j}}\left\|\phi\left(\boldsymbol{X}_{i}\right)-\boldsymbol{m}_{j}\right\|^{2}$ to be the distortion of cluster $C_{j}$, where $\boldsymbol{m}_{j}=\frac{1}{n_{j}} \sum_{l \in C_{j}} \phi\left(\boldsymbol{X}_{l}\right)$ is the cluster center and $n_{j}$ is the number of samples in cluster $C_{j}$. The cluster distortion can be then rewritten as:

$$
\begin{aligned}
d\left(C_{j}\right)= & \sum_{i \in C_{j}}\left\|\phi\left(\boldsymbol{X}_{i}\right)-\boldsymbol{m}_{j}\right\|^{2} \\
= & \sum_{X_{i} \in C_{j}} \phi\left(\boldsymbol{X}_{i}\right)^{T} \phi\left(\boldsymbol{X}_{i}\right) \\
& -\frac{2}{n_{j}} \sum_{i \in C_{j}} \sum_{l \in C_{j}} \phi\left(\boldsymbol{X}_{l}\right)^{T} \phi\left(\boldsymbol{X}_{i}\right) \\
& +\frac{1}{n_{j}^{2}} \sum_{i \in C_{j}} \sum_{l \in C_{j}} \sum_{z \in C_{j}} \phi\left(\boldsymbol{X}_{l}\right)^{T} \phi\left(\boldsymbol{X}_{z}\right) \\
= & \operatorname{tr}\left\{\boldsymbol{K}^{[j]}\right\}-\operatorname{tr}\left\{\frac{\boldsymbol{E}_{j}^{T}}{\sqrt{n_{j}}} \boldsymbol{K}^{[j]} \frac{\boldsymbol{E}_{j}}{\sqrt{n_{j}}}\right\}
\end{aligned}
$$

where $\boldsymbol{K}_{i l}^{[j]}=\phi\left(\boldsymbol{X}_{i}\right)^{T} \phi\left(\boldsymbol{X}_{l}\right)$ for $\boldsymbol{X}_{i}, \boldsymbol{X}_{l} \in C_{j}, \boldsymbol{E}_{j}$ is an identity matrix of size $n_{j}$, and $\sum_{i \in C_{j}} \phi\left(\boldsymbol{X}_{l}\right)^{T} \phi\left(\boldsymbol{X}_{z}\right)=n_{j}$.

The overall distortion for $k$ clusters can be defined as:

$$
\Omega=\sum_{j=1}^{k} d\left(C_{j}\right)=\operatorname{tr}\{\boldsymbol{K}\}-\operatorname{tr}\left\{\mathbb{E}^{T} \boldsymbol{K} \mathbb{E}\right\}
$$

where $\mathbb{E}$ is a $n \times n$ block diagonal orthonormal matrix, defined as:

$$
\mathbb{E}=\left[\begin{array}{cccc}
\frac{\boldsymbol{E}_{1}}{\sqrt{n_{1}}} & 0 & \cdots & 0 \\
0 & \frac{\boldsymbol{E}_{2}}{\sqrt{n_{2}}} & & \vdots \\
\vdots & & \ddots & 0 \\
0 & \cdots & 0 & \frac{\boldsymbol{E}_{k}}{\sqrt{n_{k}}}
\end{array}\right]
$$

To achieve effective clustering, the overall distortion in (3) needs to be minimised. Since $\operatorname{tr}\{\boldsymbol{K}\}$ is constant regardless of the clustering result, the minimisation problem reduces to maximising $\operatorname{tr}\left\{\mathbb{E}^{T} \boldsymbol{K} \mathbb{E}\right\}$ over $\mathbb{E}$. The solution of the maximisation problem can be sought by imposing the constraint $\mathbb{E}^{T} \mathbb{E}=I$, ie.:

$$
\max _{\mathbb{E}} \operatorname{tr}\left\{\mathbb{E}^{T} \boldsymbol{K} \mathbb{E}\right\} \quad \text { s.t. } \quad \mathbb{E}^{T} \mathbb{E}=I
$$

The optimisation problem in (4) is a generalised eigenvalue problem and can be solved by computing the top $k$ eigenvectors of $\boldsymbol{K}$ [3]. We note that $\mathbb{E}^{T}=\mathbb{E}$, with $\mathbb{E}^{T}$ being used to explicitly show that (4) can be seen as a generalised eigenvalue problem.

As studied in the theory of spectral clustering $[3,12,16$, 20], a normalised version of the kernel matrix is more suitable for clustering purposes. Borrowing the idea from Euclidean space, the normalised Grassmann kernel matrix is defined as $\boldsymbol{D}^{-\frac{1}{2}} \boldsymbol{K} \boldsymbol{D}^{-\frac{1}{2}}$, where $\boldsymbol{D}$ is a diagonal matrix in which the $i$-th diagonal element is equal to the sum of row $i$ of matrix $K$.

By embedding the Grassmann manifold into the new optimal space in terms of cluster distortion, any Euclidean clustering algorithm can be employed to group data. For the sake of simplicity, in the experiments we use Euclidean $k$-means to cluster the results of the embedding [12]. Figure 1 outlines the proposed clustering approach.

Input: Points $\left\{\boldsymbol{X}_{i}\right\}_{i=1}^{n}$ from the underlying Grassmann manifold.

\section{Processing:}

1: Compute the kernel matrix $\boldsymbol{K}=\left[k_{i j}\right] ; k_{i j}=k_{P}\left(\boldsymbol{X}_{i}, \boldsymbol{X}_{j}\right)$.

2: Compute the normalised kernel matrix $\widehat{\boldsymbol{K}}=\boldsymbol{D}^{-\frac{1}{2}} \boldsymbol{K} \boldsymbol{D}^{-\frac{1}{2}}$

3: Compute the top $m$ eigenvectors $\boldsymbol{u}_{1}, \ldots, \boldsymbol{u}_{m}$ of $\widehat{\boldsymbol{K}}$

4: Form a matrix $\boldsymbol{U} \in \mathbb{R}^{n \times m}$ containing $\boldsymbol{u}_{1}, \ldots, \boldsymbol{u}_{m}$ as columns.

5: Let the new set of points be $\left\{\boldsymbol{z}_{i}\right\}_{i=1}^{n} ; \boldsymbol{z}_{i} \in \mathbb{R}^{m}$, corresponding to the $i$-th row of $\boldsymbol{U}$.

6: Apply $k$-means clustering on $\left\{\boldsymbol{z}_{i}\right\}_{i=1}^{n}$.

Output: $A_{1}, \ldots, A_{m}$ where $A_{i}=\left\{j \mid z_{j} \in C_{i}\right\}$

Fig. 1: Pseudocode for the proposed Grassmann clustering. 


\section{EXPERIMENTS}

In this section we compare the performance of the proposed method against the intrinsic Grassmann clustering approach presented by Turaga et al. [18]. We first use synthetic data, followed by clustering human actions on ballet and ice skating video datasets $[4,21]$.

\subsection{Synthetic Data}

For the synthetic data, we created three clustering problems with increasing difficulty (easy, medium, hard). To create each problem, several random clusters on a specific tangent space of a Grassmann manifold were first generated. The samples of each cluster on the tangent space obeyed a normal distribution. All the samples were then mapped back to the Grassmann manifold using the exponential map [18]. By fixing the mean of each class and increasing the class variance, the clusters intertwine with each other, resulting in more difficult clustering problems.

For each clustering problem, 50 points per cluster were considered. Each problem contained either 4 or 6 clusters. Performance evaluation is measured by clustering rate, which indicates how well the formed clusters represent groundtruth clusters [1]. Briefly, the underlying measure indicates whether two given samples belong to the same cluster.

Table 1 shows the average clustering rates and the average runtimes after 50 trials for the method of Turaga et al. [18] and the proposed approach. The algorithms were implemented in Matlab on an Intel Core 2 Duo processor running at $3 \mathrm{GHz}$. Regardless of the task difficulty, the proposed approach always obtains the best results, while also being several orders of magnitude faster.

Table 1: Comparison of the clustering rate and run-time on synthetic data achieved by Turaga et al. [18] and the proposed method.

\begin{tabular}{lcclc}
\hline \multirow{2}{*}{ clusters / task } & \multicolumn{2}{c}{ clustering rate } & \multicolumn{2}{c}{ approx. run-time (seconds) } \\
& Turaga [18] & proposed & Turaga [18] & proposed \\
\hline 4 / easy & 67.61 & $\mathbf{9 1 . 9 1}$ & $6.8 \times 10^{3}$ & $\mathbf{2 . 4}$ \\
6 / easy & 70.17 & $\mathbf{8 5 . 1 4}$ & $9.6 \times 10^{3}$ & $\mathbf{5 . 1}$ \\
4 / medium & 64.25 & $\mathbf{8 2 . 0 7}$ & $6.7 \times 10^{3}$ & $\mathbf{2 . 4}$ \\
6 / medium & 67.74 & $\mathbf{8 6 . 2 3}$ & $9.4 \times 10^{3}$ & $\mathbf{5 . 1}$ \\
4 / hard & 67.89 & $\mathbf{7 9 . 2 1}$ & $6.8 \times 10^{3}$ & $\mathbf{2 . 3}$ \\
6 / hard & 67.56 & $\mathbf{8 4 . 2 3}$ & $9.8 \times 10^{3}$ & $\mathbf{5 . 2}$ \\
\hline
\end{tabular}

Table 2: As per Table 1, but using the Ballet video dataset.

\begin{tabular}{ccccc}
\hline \multirow{2}{*}{ clusters } & \multicolumn{2}{c}{ clustering rate } & \multicolumn{2}{c}{ approx. run-time (seconds) } \\
& Turaga [18] & proposed & Turaga [18] & proposed \\
\hline 8 & 73.47 & $\mathbf{8 8 . 7 5}$ & $1.2 \times 10^{5}$ & $\mathbf{1 7 . 3}$ \\
\hline
\end{tabular}

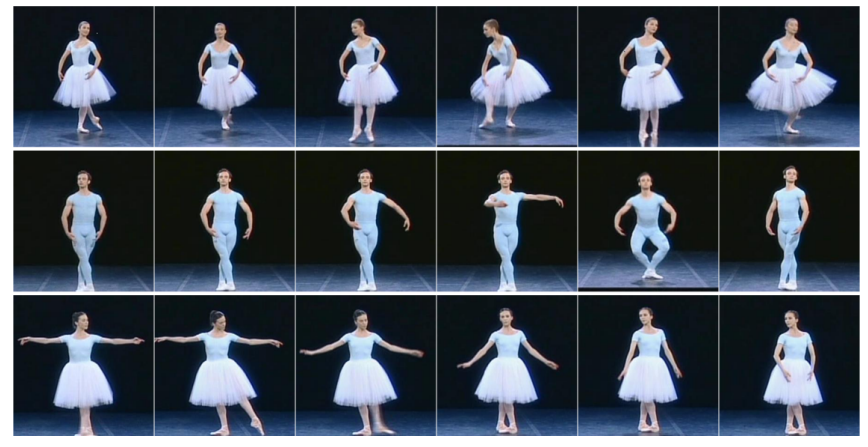

Fig. 2: Examples from the Ballet video dataset.

(a)

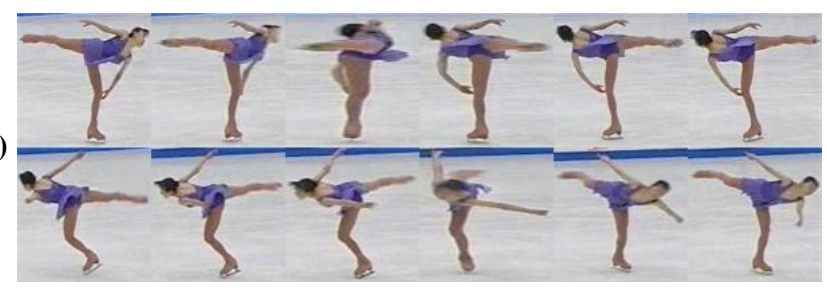

(b)

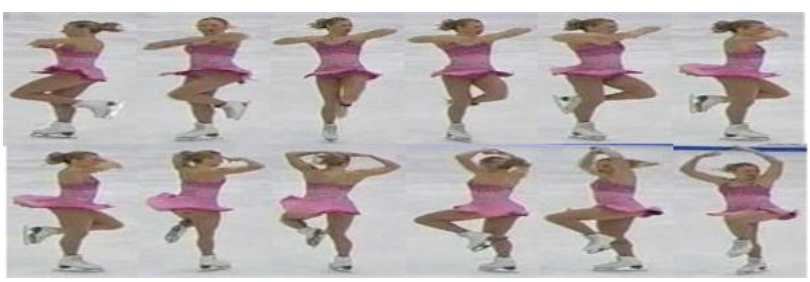

(c)



(d)

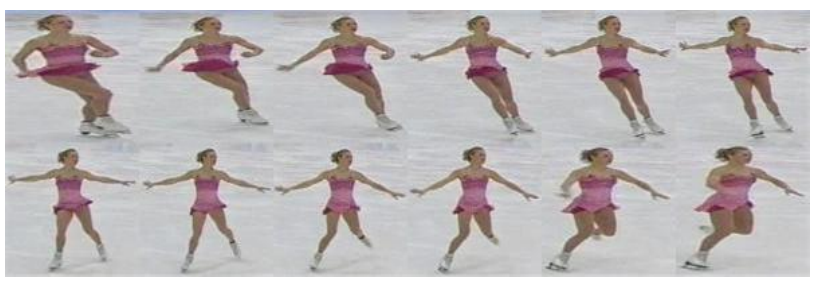

Fig. 3: Samples form the obtained clusters on the ice skating video dataset. Each row shows consecutive frames. (a) camel-spins, (b) stand-spins, (c) sit-spins, (d) gliding.

(a)

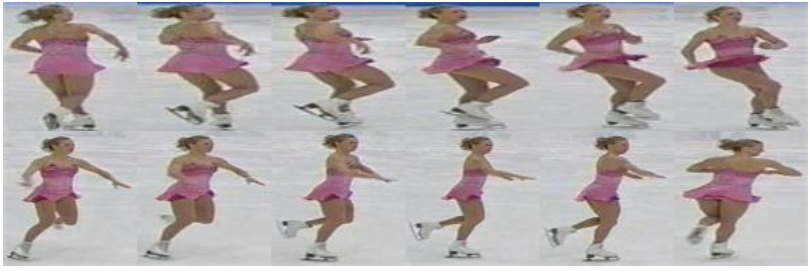

Fig. 4: Mis-clustered samples for (a) gliding, and (b) stand-spins. 


\subsection{Clustering Human Actions}

For clustering human actions, we considered a ballet dataset [4] and an ice skating dataset [21]. The ballet dataset contains 44 videos of 8 unique actions. See Fig. 2 for examples. We randomly generated 400 Grassmann points (50 points per action). We downsampled all video frames to a size of $16 \times 16$ pixels. Each Grassmann point corresponds to 6 consecutive frames from an action, meaning the clustering will done on $\mathcal{G}_{256,6}$. To generate Grassmann points, we used normalised pixel intensities as image features and generated subspaces using Singular Value Decomposition (SVD).

Table 2 shows the achieved average clustering rate and the average runtime after 50 runs. The results mirror the observations obtained on synthetic data, ie., the proposed method obtained a higher clustering rate than Turaga et al. [18], while also being considerably quicker.

The last experiment was a qualitative evaluation on the ice skating dataset. The dataset includes unconstrained videos involving real world conditions such as rapid motion of the camera and the skater. We generated 100 Grassmann points, where each point encodes 6 consecutive frames. Each frame was manually cropped (to exclude the background) and downsampled to a size of $100 \times 100$ pixels. Like in the Ballet experiment, normalised pixel intensities were used as image features and subspaces were generated by SVD. As such, the clustering problem was on $\mathcal{G}_{10000,6}$.

Fig. 3 shows several examples of the clustered actions, while Fig. 4 shows two mis-clustered examples. We note that in both cases the selected clusters are visually appealing. More specifically, Fig. 4(a) contains a small spin while the recognised cluster was gliding. Since the spin is very small in this case, the action might be considered as a hybrid of gliding and stand-spin. Fig. 4(b) shows an example of gliding while the recognised cluster was stand spin. The arm movement in this case resembles stand spins.

\section{MAIN FINDINGS AND FUTURE DIRECTIONS}

In this paper we have developed a novel approach for clustering data on Grassmann manifolds. Grassmann manifolds encode the manifold of subspaces and act as a convenient and natural medium to characterise various computer vision problems. The proposed clustering scheme embeds the Grassmann manifold into a Hilbert space where a measure of clustering distortion is minimised.

Experiments on synthetic data as well as real video data indicate that the proposed method considerably outperforms a recent intrinsic Grassmann $k$-means algorithm presented by Turaga et al. [18]. Furthermore, since the proposed approach does not require Grassmann exponential and logarithmic mappings (which are computationally very expensive), the computational cost is several orders of magnitude lower.

Future research avenues include considering clustering methods other than $k$-means when processing the embedded points. Moreover, rather than simply transferring image sets as points on the manifold, Auto Regressive Moving Average (ARMA) models can be used to represent video data [18]. Last but not least, we aim to compare our clustering approach against state-of-the-art Euclidean solutions to contrast the effect of non-Euclidean geometry.

\section{REFERENCES}

[1] H. Cetingul and R. Vidal. Intrinsic mean shift for clustering on Stiefel and Grassmann manifolds. In Proc. IEEE Conf. Computer Vision and Pattern Recognition (CVPR), pages 1896-1902, 2009.

[2] D. Comaniciu and P. Meer. Mean shift: A robust approach toward feature space analysis. Pattern Analysis and Machine Intelligence, IEEE Transactions on, 24(5):603-619, 2002.

[3] I. Dhillon, Y. Guan, and B. Kulis. Kernel k-means, spectral clustering and normalized cuts. In Proc. ACM SIGKDD Int. Conf. Knowledge Discovery and Data Mining, pages 551-556, 2004.

[4] A. Fathi and G. Mori. Action recognition by learning mid-level motion features. In Proc. IEEE Conf. Computer Vision and Pattern Recognition $(C V P R)$, pages 1-8, 2008.

[5] M. Harandi, C. Sanderson, S. Shirazi, and B. Lovell. Graph embedding discriminant analysis on Grassmannian manifolds for improved image set matching. In Proc. IEEE Conf. Computer Vision and Pattern Recognition (CVPR), pages 2705-2712, 2011.

[6] A. Jain. Data clustering: 50 years beyond K-means. Pattern Recognition Letters, 31(8):651-666, 2010.

[7] K. Jia and D.-Y. Yeung. Human action recognition using local spatiotemporal discriminant embedding. In Proc. IEEE Conf. Computer Vision and Pattern Recognition (CVPR), pages 1-8, 2008.

[8] H. Karcher. Riemannian center of mass and mollifier smoothing. Communications on Pure and Applied Mathematics, 30(5):509-541, 1977.

[9] R. Li and R. Chellappa. Group motion segmentation using a spatiotemporal driving force model. In Proc. IEEE Conf. Computer Vision and Pattern Recognition (CVPR), pages 2038-2045, 2010.

[10] Y. Lui. Advances in matrix manifolds for computer vision. Image and Vision Computing, (in press). DOI: 10.1016/j.imavis.2011.08.002.

[11] Y. Lui and J. Beveridge. Tangent bundle for human action recognition. In Proc. Automatic Face \& Gesture Recognition and Workshops, pages 97-102, 2011.

[12] A. Ng, M. Jordan, and Y. Weiss. On spectral clustering: Analysis and an algorithm. In Proc. Neural Information Processing Systems (NIPS), 2001.

[13] X. Pennec. Intrinsic statistics on Riemannian manifolds: Basic tools for geometric measurements. J. Mathematical Imaging and Vision, 25(1):127-154, 2006.

[14] A. Sanin, C. Sanderson, M. T. Harandi, and B. C. Lovell. K-tangent spaces on Riemannian manifolds for improved pedestrian detection. In Proc. IEEE International Conference on Image Processing, 2012.

[15] J. Shawe-Taylor and N. Cristianini. Kernel methods for pattern analysis. Cambridge University Press, 2004.

[16] J. Shi and J. Malik. Normalized cuts and image segmentation. IEEE Trans. Pattern Analysis and Machine Intell., 22(8):888-905, 2000.

[17] R. Subbarao and P. Meer. Nonlinear mean shift over Riemannian manifolds. Int. J. Computer Vision, 84(1):1-20, 2009.

[18] P. Turaga, A. Veeraraghavan, A. Srivastava, and R. Chellappa. Statistical computations on Grassmann and Stiefel manifolds for image and video-based recognition. IEEE Trans. Pattern Analysis and Machine Intelligence, 33(11):2273-2286, 2011.

[19] O. Tuzel, F. Porikli, and P. Meer. Learning on Lie groups for invariant detection and tracking. In Proc. IEEE Conf. Computer Vision and Pattern Recognition (CVPR), pages 1-8, 2008.

[20] U. von Luxburg. A tutorial on spectral clustering. Statistics and Computing, 17(4):395-416, 2007.

[21] Y. Wang, H. Jiang, M. Drew, Z.-N. Li, and G. Mori. Unsupervised discovery of action classes. In Proc. IEEE Conf. Computer Vision and Pattern Recognition (CVPR), volume 2, pages 1654-1661, 2006. 